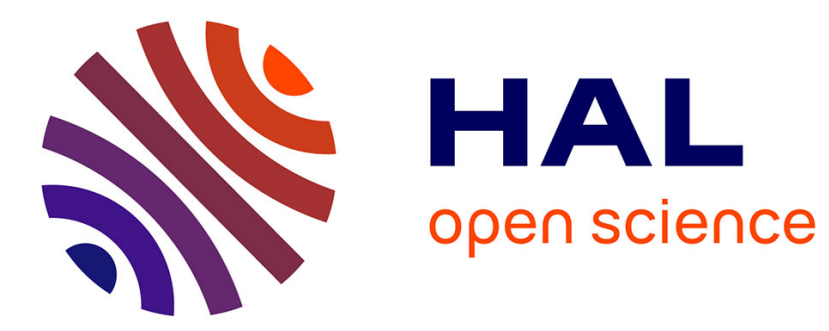

\title{
Ancêtres claniques et esprits féminins dans l'Himalaya népalais
}

Gérard Toffin

\section{To cite this version:}

Gérard Toffin. Ancêtres claniques et esprits féminins dans l'Himalaya népalais. Revue de l'histoire des religions, 1990, 207 (2), pp.159-188. 10.3406/rhr.1990.1737 . hal-00591635

\section{HAL Id: hal-00591635 \\ https://hal.science/hal-00591635}

Submitted on 13 Feb 2018

HAL is a multi-disciplinary open access archive for the deposit and dissemination of scientific research documents, whether they are published or not. The documents may come from teaching and research institutions in France or abroad, or from public or private research centers.
L'archive ouverte pluridisciplinaire HAL, est destinée au dépôt et à la diffusion de documents scientifiques de niveau recherche, publiés ou non, émanant des établissements d'enseignement et de recherche français ou étrangers, des laboratoires publics ou privés. 


\section{Ancêtres claniques et esprits féminins dans l'Himalaya népalais} Gérard Toffin

Citer ce document / Cite this document :

Toffin Gérard. Ancêtres claniques et esprits féminins dans I'Himalaya népalais. In: Revue de I'histoire des religions, tome 207, $\mathrm{n}^{\circ} 2$, 1990. pp. 159-188;

doi : 10.3406/rhr.1990.1737

http://www.persee.fr/doc/rhr_0035-1423_1990_num_207_2_1737

Document généré le 20/04/2017 


\begin{abstract}
Clan ancestors and female spirits in the Nepalese Himalayas

Clan ancestors and female spirits delimit two distinct areas of religious representations among the Tibeto-Burman populations of Nepal. The first relating to bone kinship are connected with ancestral continuity and are venerated by a specialist responsible for village cults. The second, associated with blood (or flesh) kinship, embody ambiguous values ; their priests are shamans, living symbols of otherness. Shifts sometime occur within this general framework. The Magars are notable for a conjunction between shamanism and ancestor worship. On the other hand, among the Limbus, the shaman has been rejected outside the ancestral sphere, his practices evolving towards forms of possession.
\end{abstract}

\title{
Résumé
}

Ancêtres claniques et esprits féminins délimitent deux domaines distincts dans les représentations des populations tibéto-birmanes du Népal. Les premiers relèvent de la parenté de l'os, ils sont liés à la continuité ancestrale et sont vénérés par un spécialiste chargé des cultes villageois. Les seconds, associés à la parenté de sang (ou de la chair), incarnent des valeurs ambiguës ; leurs prêtres sont des chamanes, symbole vivant de l'altérité. Ce cadre général subit parfois des inflexions. Les Magar notamment se distinguent par une conjonction entre chamanisme et ancestralité. Chez les Limbu, en revanche, le chamane se voit rejeté hors de la sphère lignagère, sa pratique évolue vers la possession. 


\section{GERARD TOFFIN}

Centre national de la Recherche scientifique

\section{ANCETRES CLANIQUES ET ESPRITS FEMININS DANS L'HIMALAYA NÉPALAIS}

Ancêtres claniques et esprits féminins délimitent deux domaines distincts dans les représentations des populations tibéto-birmanes du Népal. Les premiers relèvent de la parenté de l'os, ils sont liés à la continuité ancestrale et sont vénérés par un spécialiste chargé des cultes villageois. Les seconds, associés à la parenté du sang (ou de la chair), incarnent des valeurs ambiguës ; leurs prêtres sont des chamanes, symbole vivant de l'altérité. Ce cadre général subit parfois des inflexions. Les Magar notamment se distinguent par une conjonction entre chamanisme et ancestralité. Chez les Limbu en revanche, le chamane se voit rejeté hors de la sphère lignagère, sa pratique évolue vers la possession.

\section{Clan ancestors and female spirits in the Nepalese Himalayas}

Clan ancestors and female spirits delimit two distinct areas of religious representations among the Tibeto-Burman populations of Nepal. The first relating to bone kinship are connected with ancestral continuity and are venerated by a specialist responsible for village cults. The second, associated with blood (or flesh) kinship, embody ambiguous values; their priests are shamans, living symbols of otherness. Shifts sometime occur within this general framework. The Magars are notable for a conjunction between shamanism and ancestor worship. On the other hand, among the Limbus, the shaman has been rejected outside the ancestral sphere, his practices evolving towards forms of possession. 
Comme l'a mis en évidence Claude Lévi-Strauss dès 1947, l'opposition entre parenté de l'os (rus) et parenté de la chair (sha) joue un rôle central parmi les populations de l'Himalaya et de la Haute Asie'. Au Tibet, l'enfant, pense-t-on, est fait des os que lui donne son père et de la chair (ou du sang) qui lui vient de sa mère ${ }^{2}$. La parenté de l'os est censée passer du père aux enfants par l'intermédiaire du sperme, celle de la chair par les sécrétions vaginales de la mère ${ }^{3}$. La première évoque l'idée de permanence - le clan patrilinéaire lui-même est appelé ru [rus], " os ", en tibétain -, la seconde apparaît plus éphémère, transitoire, à l'instar du cadavre humain dont la chair se décompose rapidement alors même qur les os restent imputrescibles. Ces deux catégories complémentaires, qui définissent l'identité des individus et qui renvoient peut-être originellement à un symbolisme sexué des couleurs (sperme et sang menstruel), correspondent également à quelque chose de très profond parmi les peuples de langues tibéto-birmanes du moyen pays népalais. Chez les Tamang de l'Est par exemple, la filiation de l'os (nakhu), par laquelle s'héritent les statuts, les droits sur la terre, l'appartenance au groupe, s'oppose à la filiation de la chair (shya), par laquelle se transmettent le sang,

1. Lévi-Strauss (1947, chap. XXIII et XXIV). Je remercie Corneille Jest pour ses précieuses remarques faites sur une première version de cette étude.

2. Par exemple R. Stein (1962, 70). Pour le Ladakh, voir P. Dollfus (1989, 212) ; pour les populations tibétaines du Népal : C. Jest (1975, 241) et N. Levine (1988, chap. III). En tibétain, la parenté par le sang se dit t'ak [khrag] (Jäschke, $1949,49)$. 36).

De telles conceptions existent également en Inde; cf. A. Padoux (1984,

3. N. Levine $(1988,58)$, P. Dollfus $(1989,212)$. Pour les Lepcha du Sikkim, cf. G. Gorer $(1938,289)$, cité par Lévi-Strauss. Selon les traités de médecine tibétaine, l'embryon est constitué à partir du sperme masculin et du sang féminin (F. Meyer, 1981, 111). Dans un mythe cosmogonique tibétain, le sang du klu (être aquatique et chthonien) féminin primitif [dont le cosmos est sorti] donne naissance aux cinq océans, de sa chair nait la terre et de ses os les montagnes; cf. P. Kvaerne (1981, 250). 
la chaleur et, au dire de certains, le "souffle "4. Les Tamang de l'Ouest, cux, suffixent lf mot shya, "chair ", à des termes de parenté pour indiquer le sexe féminin (ex. : gren-shya : "femme aînée ") ou à de petits groupes de parents composés de femmes (ex. : leng-shya : parents nés d'une amitié rituelle entre deux femmes). Ios déterminants peuvent parfois changer, comme chez les Magar et les Kulung Rai qui qualifient le còté maternel de parenté du lait, mais partout l'opposition entre ces deux héritages familiaux se fait sentir. Le premier (os) ressortit davantage au monde social, ordonné, le second (sang) au monde physiologique. " naturel ". En outre, dans nombre de populations népalaises, l'inceste, source de segmentation à l'intéricur du clan, est appelé "briser l'os " et les cérémonies visant à rétablir l'ordre après une telle transgression : "restaurer la relation de l'os $"$ ".

Selon Lévi-Strauss, rette dichotomie serait liée à l'échange généralisé qu'engendre le mariage prescriptif avec la fille du frère de la mère. En réalité, l'opposition de l'os et de la chair est présente dans plusieurs types de sociétés de l'Himalaya : les unes pratiquent l'échange restreint, d'autres l'échange généralisé, d'autres encore n'ont pas de système matrimonial prescriptif. Les termes " os " et "chair " s'appliquent donc: autant à des individus, tels le père et la mère qui contribuent chacun à la formation de l'enfant, qu'à des groupes entiers de parents. On peut bien sûr postuler que là où subsiste la bipartition en question le système matrimonial appartenait à époque ancienne au type échange

4. B. Steinmann $(1987 a, 187)$.

5. Informations recueillies par l'auteur dans le massif du Ganesh Iimal. Pour une présentation du systeme de parenté des Tamang de l'Ouest, cf. G. Toflin (1986). A propos des Gurung, voir aussi B. Pignède (1966, 336).

6. Pour les Magar, cf. M. Oppitz $(1983,223)$ et $(1989,58-62)$ ainsi qu'A. de Sales (1985, 66). Pour les Kulung Tai, voir (. Mac Dougal (1973, 212). Par métaphore, la lignee maternelle est appelée nwi (ou nui), "lait ", chez les Kham Magrar (de Sales, 1986, 66).

7. Cf. par excmple C. Mac Dougal (1973, 211), P. Sagant $(1982,172)$ et M. Oppitz (1983, 223). Semblablement, chez les Kulung Rai, tout mariage avec un parent utérin dans un degré de parenté trop rapproché, donc incestueux, est appelé dudh phora, "casser le lait (C. Mac Dougal, 1973, 215). 
généralisé. Mais outre que cette thèse n'est guère démontrable du point de vue historique, certains auteurs défendent une opinion inverse; pour eux, c'est l'échange restreint, le mariage répété entre cousins croisés bilatéraux, qui serait chronologiquement premier dans les collines himalayennes (Allen, 1975). Il n'est pas possible ici d'entrer dans cette discussion, fort complexe. Pour l'heure, retenons seulement qu'aucune correspondance directe ne peut être mise en évidence entre la " théorie de l'os et de la chair " et la structure sociale matrimoniale.

Nous laisserons donc de côté la logique pure du système de parenté, quitte à revenir sur certains aspects de l'alliance, pour nous intéresser au traitement symbolique de l' "os" et de la "chair ". Il s'agira plus précisément d'analyser les analogies que les populations himalayennes ont tissées entre cette opposition, fondée sur la distinction entre les sexes, et les représentations religieuses. La parenté de l'os et celle de la chair circonscrivent en effet deux domaines autant fondamentaux qu'exemplaires : celui de la parenté masculine et celui de la parenté féminine (ou encore : de la filiation agnatique et de l'alliance), autour desquels s'articulent de nombreuses croyances et pratiques religicuses. Elles délimitent aussi deux catégories : ancêtres claniques d'un côté, esprits féminins de l'autre, qui jouent un rôle clef, quoique mal apprécié jusqu'ici, dans les religions himalayennes. Pour en faire la démonstration, on se limitera aux populations tribales népalaises de langues tibéto-birmanes, lesquelles, des Magar à l'ouest aux Limbu à l'est, vivent toutes dans le pays des collines entre 1500 et $2500 \mathrm{~m}$, au pied de la haute chaine. Elles constituent un ensemble à la fois suffisamment homogène et différencié pour y déployer avec quelque sécurité une analyse comparative. Précisons pour l'intelligence du texte qui va suivre que ces sociétés sont toutes patrilinéaires et possèdent toutes des groupes d'unifiliation - plus structurés et profonds à l'ouest et au centre qu'à l'est. Elles reconnaissent aux hommes un 
rôle dominant dans l'organisation socio-politique et relèguent délibérément l'espèce féminine aux confins de l'espace socioreligieux. Leur économie dépend de l'agriculture et de l'élevage, mais certaines d'entre elles accordent encore à la chasse une place non négligeable.

Une telle dualité permet, entre autres, de mieux comprendre la coexistence au sein de ces groupes ethniques de deux types de spécialistes religicux : le prêtre qualifié généralement de "tribal ", mais qu'il convient plutôt d'appeler "clanique ", et le chamane. La bipolarisation entre ces deux prêtres constitue, on le sait, un trait récurrent parmi les populations tibéto-birmanes des collines népalaises ${ }^{8}$. Le premier s'occupe du monde surnaturel ordonné, il est associé à la continuité ancestrale et aux groupes de patrifiliation, souvent par l'intermédiaire du territoire villageois. Le second est en charge des forces de désordre qui affectent les individus; il dévoile les aspects ambigus de l'univers et peut officier dans n'importe quel clan, parfois même auprès de personnes ètrangères à son groupe ethnique. Le prêtre clanique a des affinités avec le haut et célèbre ses rituels de jour. Le chamane, lui, relève du bas et officie généralement de nuit. Or ces deux spécialistes sont très marqués du point de vue de l'ascendance. Ainsi, chez les Limbu du Népal oriental, le prêtre clanique phedāingmā se recrute plutôt en ligne paternelle alors que le chamane est élu en ligne maternelle (Sagant, 1976, 71). D. Holmberg $(1983,44)$ nous apprend par ailleurs que les Tamang mettent en rapport la vie harassante des femmes avec celle, semée d'embûches et pleine de détresse, des chamanes. En bref, les prêtres claniques s'opposent aux chamanes comme la parenté de l'os à la parenté de la chair. Et lorsque s'observe, dans certains cas particuliers, un chevauchement ou un cumul des fonctions sacerdotales, les catégories de l'os et de la chair continuent toujours d'ordonner les représentations religieuses.

8. Sur cette question, citons parmi beaucrup d'autres A. Fournier (1974), N. Allen $(1976 b, 512-521)$ et S. Strickland $(1982,49-50)$. 
Prêtre clanique el ancêtres agnatiques

Le prètre clanique, par définition, est attaché à un clan particulier et appartient à cette unité de parenté. Véritable mémoire du groupe, il maintient vivant le répertoire mythique et permet de la sorte à la société de préserver son ètre culturel. Expert en généalogie, il sert de trait d'union entre le temps primordial des origines et le temps présent. Toutefois, le lien avec les ancêtres est plus ou moins accentué selon les cas et prend des formes différentes. Il est très fort dans le Népal oriental. Le dewa dhämi des Thulung Rai par exemple célèbre le culte des ancêtres (à distinguer du culte des morts) à dates fixes et introduit, lors des rituels de mariage, la nouvelle épouse auprès des ancêtres (pitri) de la famille du mari (Allen, 1976b, 511). Chez les Tamang du Népal central en revanche, la relation, bien que présente, se fait moins nette. Parmi ceux qui vivent à l'est de la vallée de Kathmandou, on peut distinguer deux types de clans selon qu'ils font appel à des lamas ou à des prêtres claniques pour le culte des divinités tutélaires du clan. Les premiers vénèrent des dieux du panthéon lamaïste et lisent le Ruichen Cyopge, un texte de prière écrit en tibétain. Les seconds propitient les fondateurs de leur clan en récitant le livre Doñrap écrit en devanagari10. Selon Brigitte Steinmann $(\mathbf{1 9 8 7 b})$, les clans du second groupe seraient plus proches de la religion archaïque prébouddhiste. Le remplacement des aïeux originels par des figures divines explique peutêtre le relatif effacement du culte des ancêtres chez les Tamang. Parmi les membres de cette ethnie établis à l'ouest de la vallée de Kathmandou, les divinités claniques, temba la, kulgi la ou dimgi la, ont perdu leurs attaches ancestrales. Elles sont considérées comme de simples figures du pan-

9. Chez les Limbu, c'est également le prêtre clanique qui vénère les ancêtres ; cf. P. Sagant (1976, 58-60).

10. Cf. B. Steinmann $(1987,310)$. Doñrap vient peut-être du tibélain gduńrabs, "génération ", d'après C. Jest. 
théon bouddhiste (ou hindou), éventuellement comme les mânes d'anciens lamas renommés pour leur sainteté. On ne les propitie qu'à échelle familiale réduite, à l'intéricur de chaque maisonnée, jamais collectivement. Le souvenir des ancêtres reste pourtant présent ģà et là, même s'il n'est jamais possible de parler d'évhémérisme. Ainsi les Ghale du massif du Ganesh Himal, un petit groupe ethnique autonome mais fortement tamangisé, appellent meme, "grandpère ", "ancêtre ", le coq blanc qui personnifie leur divinité clanique. Chez les Tamang de l'Ouest par ailleurs, le prêtre clanique lambu oflicie toujours à côté du poteau central de la maisonnéc, siège des mânes familiaux, et attache deux fois par an à ce pilier la branche de châtaigner qui lui sert à invoquer les esprits au cours des rituels (Toffin et al., 1986, 108-109). De façon significative, le lien entre prêtre clanique et ancêtres du groupe est parfois explicitement rapporté à la parenté de l'os. Chez les Thulung Rai, le mot pour " ancêtre " (seor) dérive peut-être lui-même de ser (ou seser) qui désigne l'os (Allen, 1976a, 268).

Les prêtres claniques desservent également les dieux du terroir, ceux dont le groupe s'est approprié la terre et entre les mains desquels repose la prospérité des habitants. Ils incarnent le lien avec le sol nourricier qui porte villages et récoltes. Toutes les sociétés considérées lient en effet la stabilité de la société humaine à une stricte délimitation du territoire et à l'organisation d'un espace clos, borné. L'individu n'est pas seulement défini par sa position au sein du groupe de filiation, mais aussi par son appartenance à un village particulier, ’̀ une communauté de sol. Chez les Tamang de l'Ouest, le lambu (ou yenden aba) vénère les dieux du terroir, yulgi la ou sibda neda, deux fois par an : en octobre-novembre et en mai-juin. Le culte se déroule en divers points du territoire, surtout dans les parties surplombant le village. Ces divinités sont aussi propitiées par les prêtres claniques lambu à l'occasion de grandes fêtes dansées villageoises, au cours desquelles la communauté 
réaffirme son ancrage dans la terre qu'elle occupe et renouvelle par là même son unité. Ainsi, le mane chyaba que les Tamang du Ganesh Himal célèbrent durant la saison froide, débute par des danses des dieux du sol incarnés par des jeunes gens de la localité. Ces danses, organisées sur la base de chaque village, de chaque hameau sont coordonnées et dirigées par le lambu. On retrouve chez les Gurung des faits similaires : cinq fois dans l'année, le mardi le plus proche de la nouvelle lune, le prêtre clanique hlevri officis au nom de la communauté villageoise un rituel en hommage aux dieux chthoniens silde naldo. Il leur demande de faire tomber le plus tôt possible les premières pluies de mousson et de protéger le village. L'un des dix cônes de riz qu'il modèle à cette occasion symbolise le dieu du sol lui-même. Ces rituels s'accompagnent de récitations, de prières, de sacrifice de poisson, de coq et d'oiseau sauvage, et font l'objet de grandes festivités (Pignède, 1966, 306-307). Une telle association entre génies locaux et prêtre clanique est également à l'œuvre dans tout le Népal oriental, notamment autour du culte aux dieux bhume, du nép. bhuĩ : "sol " (Allen, 1981).

Un point mérite d'être souligné : ancestralité et territorialité sont toujours associées. Tous les groupes tribaux des collines népalaises rattachent d'une certaine façon le territoire qu'ils occupent et dont ils tirent leur subsistance à l'ancestralité. Tous ont des mythes de fondation qui retracent la manière dont l'espace original du groupe en question a été choisi, divisé, peuplé par les ancêtres fondateurs. Ces récits, indéfiniment répétés, acquièrent une réalité sociale et une efficacité symbolique dont la société des vivants ne saurait se passer. Ils constituent un arrière-plan symbolique toujours présent et un point de référence permettant aux unités familiales de se situer spatialement les unes par rapport aux autres, jusque dans le plus petit des hameaux. C'est à travers les hauts faits de ces héros défunts que l'existence sociale prend aux yeux des vivants sens et valeur. Les 
Gurung sont ceux qui ont poussé le plus loin le rapprochement. Nous savons aujourd'hui, grâce à S. Strickland (1983), que l'âme du mort, plah, est composée de deux éléments dans les représentations collectives propres à ce groupe ethnique : un élément bénéfique, appelé plah proprement dit, qui est guidé lors des rituels funéraires jusqu'au pays des morts, situé au nord, au-delà des limites du pays gurung, et un élément malintentionné, un mauvais mort démoniaque connu sous le nom de $m \tilde{o}$, du terme générique employé pour désigner les démons, qui, lui, s'incorpore au territoire tribal et vient s'agréger à une divinité du sol. Ces aspects maléfiques $m \tilde{o}$ s'opposent aux ancêtres khewa; ils s'attaquent aux humains, volent leurs âmes et boivent leur sang. Ils n'en définissent pas moins une certaine figure de l'ancestralité. C'est sans doute à cette croyance qu'il faut rattacher le mythe de Karabare, chasseur de miel sauvage, souvent cité dans la littérature ethnographique ${ }^{11}$. Le mythe, que nous simplifions ici, raconte l'histoire d'un homme, Karabare, qui part à la recherche de miel d'abeilles sauvages en compagnie de ses sept fils. Arrivés au sommet d'un précipice d'où il faut dérouler une échelle de corde pour approcher les essaims, les fils décident de tuer leur père en vue de prendre sa jeune femme. Leur plan échoue. Pour les punir, Karabare les transforme en neuf êtres démoniaques qui, dispersés en neuf points différents, correspondent chacun à une divinité et à un village particulier de la société gurung d'aujourd'hui. La relation entre ancestralité et territorialité passe dans ce cas précis par les divinités gardiennes du sol. La divinité tutélaire Kyolõ Thankhu, qui réside dans une forêt sacrée, non loin du village, légèrement sur les hauteurs, est d'ailleurs classée tantôt dans la catégorie des dieux du sol, tantôt dans celle des " rois des morts ", shin mru (Strickland, 1982, 54).

11. Sur ce mythe, voir S. Strickland (1982, 152-176 et 182-185) d'après des manuscrits non publies de Pignede. On retrouve eette histoire chez les Magar de la Kali Gandaki ; cf. C. Jest (1976, 299-300). Les Thulung Rai possèdent également un mythe de collecteur de miel sauvage, mais sa signification semble assez différente (Allen, 1976a, 229-231). 
En s'agrégant au pays habité, la composante démoniaque de l'homme, mõ, tout le pandémonium au sens étymologique du terme en fait, entre en contact avec les divinités telluriques protectrices. Cette fusion explique l'ambivalence fondamentale de ces puissances divines ot leurs aspects maléfiques (Toffin, 1987).

Les Tamang eux aussi réfèrent le territoire habité, cultivé, aux ancêtres. C'est ce qui ressort d'un rituel appelé thabsyang, célébré par un lama bouddhiste sur les bords d'une rivière en cas de naissance d'un enfant illégitime. Les bâtards nés d'un père inconnu menacent le village car ils ont pu etre engendrés par deux personnes appartenant à un même rlan ou par une femme tamang et un intouchable. Pollués par une telle union, les dieux du sol peuvent faire abattre des catastrophes, notamment de la grêle, sur le village. Le rituel thabsyang (du tib. thab : "foyer ", siège des ancêtres familiaux ?) vise justement à pacifier ces divinités et à purifier les parents de l'enfant avant la naissance ${ }^{12}$. On le: voit bien : les dieux du terroir sont sensibles aux désordres matrimoniaux qui enfreignent les règles d'exogamie clanique et menacent de casser l' "os " du clan. Qu'un lama, et non un prêtre clanique, dirige le rituel n'infirme pas le rapport établi, car il semble bien que le premier se soit progressivement attribué certaines fonctions dévolues autrefois au lambu ${ }^{13}$.

Le prêtre clanique joue donc un rôle clef dans les réseaux villageois : en lui se conjuguent autochtonie et ancestralité. C'est la raison pour laquelle il est si étroitement associé au pouvoir local : on le choisit souvent parmi les proches d'un chef de clan, parfois même parmi ses frères, et son

12. Informations recueillies dans le Ganesh Himal. Sur ce rituel, voir aussi A. Höfer $(1981,30)$.

13. C. Jest me signale que les Tamang de Kakani (Nuwakot) commeneent à faire appel à des lamas pour célébrer leurs cultes lignagers à la place du prêtre tribal, appelé localement laptaba. Sur les rapports entre prôtre clanique et lama chez les Tamang, cf. D. I Iolmberg (1989). Chez les Limbu du Népal oriental, c'est le prêtre clanique phedãingmä qui est chargé d'écarter la grêle (Sagant, 1976. 58), 
emploi est héréditairc. L'ancestralité au demeurant se définit toujours en termes politiques : le clan fondateur du village. occupe une fonction dominante dans la localité et détient généralement la charge de chef de village. C'est lo prêtre attaché à ce clan qui préside les cultes territoriaux importants et qui agit au nom de la communauté tout entière. I ui scul a le droit de propitier les divinités du sol les plus anciennes, les plus puissantes, celles associées à la création du village. Chez les Tamang, le prêtre lambu récite en ces occasions la liste des ancêtres de son groupe agnatique, de leur point de départ, situé au 'Tibet, jusqu'au point d'arrivé actucl, ou vice versa, selon les moments de la cérémonic. De cette manière, tous les ans, le clan retrace l'itinéraire de sa migration originelle et redonne vie aux éléments dont cot composée son ancestralité. Ce passé, récité et revécu, définit et justifie le clan en question dans sa position prééminente vis-à-vis des autres groupes de parenté de la localite. Dans ces rituels, le prètre clanique apparait commr le, romplément sacerdotal du chef de village'14.

On retrouve une telle ronjonction prètrise locale-pouvoir rhez les (iurung. Pignède (1966, 296-297, 302) avait, déjà relevé que les prêtres claniques hleuri (klihbri) se: prévalaient d'un lien particulier avec les anciens rois locaux pré-gorkha connus sous le nom de Ghale. Eux seuls ont le droit de célébrer les funérailles de ces ex-familles royales (ibid., 296). Let mot hlevri vient peut-être même du mot hle qui signifie "roi" en gurung et qui désigne les (ihale dans la langue locale. Pignède avait aussi suggéré que le rulte des dieux du sol devait etre rattarhé à l'ancienne. organisation politique de la société gurung. Au rours des fêtes dédiées à ces divinités, notait-il, le hlewri et to chef de village agissent toujours de (oncert (ibid., 307). Le second

14. 1). IIrlmbre $19 \times 0,240$ indiqur qu'il existe dans son village d'etude un prêtre clanique communal dont la charge passe hériditairement à l'intérieur d'un mème patrilignage sans doute le patrilignage dominant: te pritre, appule kuingyam lambn, priside aux cultrs villageois. 
pourvoit à la plupart des offrandes destinées aux ancêtres et aux dieux hleh (kleh), " maitres ". Notons cependant qu'ici comme chez les Tamang, le lamaïsme menace directement le prêtre clanique : c'est dans les régions où il y a le moins de lamas que les hlewri sont les plus nombreux et inversement (ibid., 296).

\section{Chamanisme et esprits féminins}

Le chamane appartient à un monde différent, sans rapport aucun avec les patriclans, les chefs et les héros mythiques. La séparation avec l'univers agnatique est telle que cet intercesseur se tient généralement à l'écart des ancêtres protecteurs du groupe social. Chez les Thulung Rai par exemple, il lui est défendu d'entrer en contact avec les pierres du foyer, incarnation des figures ancestrales (Allen, $1976 b, 514)$. Cet évitement fait penser aux nombreux interdits qui touchent les femmes. Car ces dernières, elles non plus, ne doivent pas approcher l'autel des ancêtres et sont les premières à souffrir en cas de transgression de l'interdit (Steinmann, 1987a, 130-131). On a là une homologie structurale importante : les chamanes sont au genre féminin ce que les prètres claniques sont aux unités de patrifiliation dominées par les valeurs masculines. Il n'est d'ailleurs pas rare que les femmes soient elles-mêmes chamanes (un prêtre clanique est toujours de sexe masculin) et l'on sait que le costume chamanique comporte certains éléments typiquement féminins : large jupe et collier en bandoulière notamment ${ }^{15}$.

Nous voudrions étayer cette thèse par deux observations tirées des Tamang de l'Ouest. Comme l'a montré Holmberg $(1983,45)$, les chamanes sont en étroite relation avec

15. Cif. D. IIolmberg (1983, 44;. A propos des chamanes poju (= pucu) gurung, B. Pignede (1966, 296) ecrit : "I.e pucu porte souvent son collier en bandoulière pour rappeler le poro (ou pro), vêtement que portent les femmes sur leur corsage et qui est attache sur une épaule. " 
des esprits particuliers appelés tsen. Ces esprits, qui font penser aux divinités tibétaines btsan de l'espace intermédiaire (associées à la couleur rouge), jouent un rôle important dans les ritucls chamaniques. Les chamanes les considèrent comme des dieux tutélaires au même titre que less lente, esprits des chamanes morts. C'est grâce à eux qu'ils acquièrent une vision de l'au-delà dont les autres humains sont privés. Un chamane sans tsen, dit-on, ne peut exercer pleinement son art. Or ces tsen sont des esprits féminins. Plus encore: : ils s'héritent en ligne maternelle. Sous la forme de fleurs, mhendo, particulières, ils passent de mère en fille, contribuant par leur présence bienveillante à la prospérité, la force et le bien-être d'une maisonnée. Ils se transmettent au moment du mariage dans les bijoux donnés par la mère à sa fille. Les tsen aiment les bijoux précieux, lor et l'argent. Ils ont une prédilection pour les fleurs, celles de couleur rouge surtout, dans lesquelles ils vivent et dont ils se nourrissent. Médiateurs entre le ciel et la terre, ils se trouvent dans la même position que les femmes qui servent d'intermédiaires entre les groupes échangistes de la société tamang et ont des droits et des obligations dans deux clans simultanément (Holmberg, 1983, 45). Les familles dotées de tels esprits doivent faire appel à un chamane tous les ans ou tous les trois ans, au printemps ou a l'automne, pour les pacifier. L'intercesseur leur offre des fleurs, de rhododendrons par exemple, et revigore la force de vie des nembres de la maisonnée, surtout de la maîtresse du logis. Seulement ces esprits sont fort ambivalents : ils peuvent se muer en etres malfaisants s'ils s'estiment mal ou insuffisamment vénérés. Ils peuvent rendre aveugles les hommes et rendre stériles les femmes en suçant leur sang menstruel comme ils sucent le nectar des fleurs - deux troubles physiques souvent associés dans la nosologie populaire des populations de l'Himalaya. Un chamane est alors convoqué. Pour identifier la "fleur " de la femme, il énumère toutes les fleurs qu'il connait. Sa transe débute dès qu'il prononce celle à 
laquelle sa patiente est attachée (Höfer, 1974, 178). Quand on sait que les femmes sont liées aux fleurs dont elles se décorent la tête au moment des fêtes et dont les bijoux imitent la forme, et que leur sexe est appelé métaphoriquement mhendo, "fleur ", on entr'aperçoit un vaste système symbolique unissant nature, féminité et esprits surnaturels. Par l'intermédiaire des chamanes se trouvent ainsi constituées des chaînes génésiques spécifiques où les valeurs ambiguës de la femme, celles liées à ses fonctions de sexualité et de procréation, s'affirment. Ces chaînes féminines, incertaines, inconstantes, quasi mystiques, s'opposent à la parenté masculine de l'os où dominent les règles de transmission régulières, préétablies.

Le second élément de la culture tamang qu'il convient de riter a trait à un autre type d'esprit particulier : Kale ama, qui occupe une place centrale dans les représentations liées à l'enfance. Jusqu'à l'âge de douze ans en effet, garçons -t filles vivent entièrement sous la coupe de cet esprit féminin. 'Tout nouveau-né, disent certains, a quatre mères : sa mère humaine " nourricière " et trois Kale ama. Qu'elle soit une ou triple, Kale ama contribue à l'affermissement et à la gestation de l'être physique et social de l'enfant. Elle construit son corps, dit-on, comme les abeilles bâtissent leurs essaims, butinant de fleur en fleur, dans de vastes prairies situées dans la partie supérieure de l'univers. La force de vie (tam. : so) de l'enfant - d'autres disent son âme (bla) a elle-même la forme d'une fleur (so mhendo), autrement dit d'un vagin, et dépend entièrement du bon vouloir de rette puissance surnaturelle. (ie n'est qu'à l'âge de douze ans que cette forme de vie externo, cette âme-fleur, s'incorpore à un arbre et se transforme en une bla-sing (ou so dungma), l' "âme-arbre " à laquelle tout homme adulte est mis en rapport. L'enfant devient à ce moment-là une personne: indépendante. $\mathrm{Or}$, là aussi, le chamanisme apparaît essentiel. Car cette figure énigmatique entretient avec l'enfant des relations paradoxales. Bien qu'elle fasse partie intégrante 
de la petite personne, qu'elle entre dans son identité la plus profonde de la manière la plus positive qui soit, Kale ama peut porter préjudice à l'enfant qui lui est lié et le rendre malade en retenant son "âme " prisonnière. Les parents convoquent aussitôt un chamane (bompo) qui part à la recherche de cette force de vie, Kali ama sangba ou Kali ama khyonba, ici totalement identifiée à l'esprit féminin. Grâce à ses pouvoirs magiques, le chamane se transporte dans les sphères célestes et cherche dans les étendues fleuries qui s'étendent sous ses yeux la fleur qui correspond à l'enfant malade. Deux signes la lui font reconnaître : la fleur est flétrie et il entre en transe dès qu'il marche à proximité. Puis il ramène Kale ama, la force de vie de l'enfant, sur terre, et la réintègre dans le corps de son patient, selon une technique courante dans les séances chamaniques organisées à l'intention des adultes. Ie chamane intervient en outre lors du rituel de passage appelé kra bre ba qui marque la fin des douze premières années du garçon et son entrée dans l'état adulte. Pour une fille, le rituel chamanique de cloture intervient à l'âge de sept ans. Dans les deux cas, la cérémonie rompt la dépendance de l'enfant vis-à-vis de Kale ama.

Le rôle du chamane vis-à-vis des esprits féminins peut ètre précisé grâce à un mythe tamang rapporté par Holmberg (1983, 52-53) :

A l'origine, les tsen et les humains n'étaient pas séparés. Ils vivaient dans le même monde et s'intermariaient. Né de là lleur d'un arbre cosmique, Gesere Tsen maria sa fille, Tingtsa Rani, i un homme appelé $m h \bar{a}$, " mari de sceur ". Un jour, Mha partit $\dot{A}$ la chasse avec le frère de Tingtsa Rani, shyangpo, "frère de femme ". Shyangpo monta sur une colline suivi de son chien afin de rabattre le gibier. Mha resta en arrière, caché dans l'anfractuosité d'un rocher, prêt à capturer le gibier. Un petit oiseau vola. Mha l'attrapa et le cacha dans ses vêtements. Lorsque Shyangpo redescendit, Mha lui annonça qu'aucun gibier n'était passé. Mais le chien de son beaufrère gesticulait et aboyait rageusement autour de lui. "Quelque chose a dû venir si j'en crois mon chien ", dit Shyangpo. Mha montra alors le petit oiseau qu'il avait attrapé : "Cie doit être ceci. "Ils divisèrent le volatile et Shyangpo dit à son compagnon de mettre une part de côté pour sa sreur. Ils regroupèrent ensuite la viande : elle 
était si lourde qu'ils durent la fixer à une perche et la porter à l'épaule. Rentré chez lui, Mha dit à sa femme qui était en train de tisser : "Tiens, voilà un cadeau que t'envoie ton frère. "Et il jeta le morceau de viande sur la cuisse de Tingtsa Rani. Mal lui en prit : ce qui n'était pour lui qu'un petit oiseau représentait pour les tsen un gibier de grosse taille. Aussi son geste brisa net la jambe de son épouse. Gesere Tsen entra dans une violente colère et ordonna à sa fille de quitter son mari sur-le-champ. 'Tous les tsen partirent et gravirent une colline au sommet de laquelle ils construisirent une barrière avec des branches et des pierres. "Peux-tu nous voir d'où tu es ?" crièrent-ils à Mha. "Rien qu'un peu ", répondit ce dernier. Ils allèrent alors plus loin et recommencèrent. "Et d'ici, nous vois-tu ou non ?" "Guère ", répondit Mha. Les jours suivants, les tsen montèrent de colline en colline, demandant à chaque fois si Mha pouvait les apercevoir. Après avoir franchi neuf collines ils construisirent une dernière barrière. Cette fois-ci, ils étaient trop loin et Mha ne put les voir. Gesere 'Tsen lui dit alors : "Maintenant nous sommes séparés. Tu engendreras le lignage des hommes; quant à nous, nous rejoignons celui des dieux. Nous nous nourrirons du suc de rhododendrons et nous vivrons dans leurs fleurs. Si vous nous traitez convenablement, si vous nous faites des offrandes appropriées, toi et tes descendants. la richesse, la force et la prospérité seront votre lot."

Ce mythe, dont nous ne pouvons aborder ici tous les détails, nous permet de passer du plan proprement religieux, celui du monde des esprits, à un plan social, plus précisément matrimonial, et d'élargir le champ des attributs symboliques associés au chamane. Il n'est certes pas question d'intercesseur dans cette rhasse malheurruse. Deux types d'acteurs, et deux seuls sont en présence : d'un côté less humains en position de preneurs de femmes, de l'autre, les esprits tsen en tant que donneurs de femmes ${ }^{16}$. Pourtant, la référence au chamanisme est transparente. Elle ressort tout particulièrement : $1^{\circ} \mathrm{du}$ lien avec les tsen, qui sont au cour du récit; 20 du mariage avec lesdits esprits (condition

16. On retrouve la mêne association entre donneurs de femmes et monde des esprits, surtout malfaisants, chez les (iurung. S. Strickland (1982, 246) donne l'exemple des rituels funéraires pendant lesquels les "oncles maternels * ashó sont placés à l'ouest de la maison du défunt, direction du soleil déclinant. et de la mort. A la fin de la cérémonie funèbre, les "oncles maternels " sont par ailleurs chassés vers l'ouest de la même manière que les démons à la fin d'un exorcisme. D'une façon générale, les populations de langues tibéto-birmanes du Nepal considèrent les démons comme des êt res féminins. 
nécessaire pour devenir chamane dans beaucoup de sociétés, notamment sibériennes); $3^{\circ}$ de la chasse dont on connait l'importance dans l'imaginaire de cette institution religieuse. On est donc en présence d'un mythe travesti, crypté, qui en dit beaucoup sur le chamanisme sans jamais le citer directement. Mettons les points sur les $\mathrm{i}$ : le récit ne fait que traduire certains traits fondamentaux du chamanisme dans un code ressortissant à l'alliance. Structurellement, le chamane se trouve dans la même position vis-à-vis des esprits tsen que le gendre du mythe tamang : c'est du mariage avec ces esprits, dont il partage à bcaucoup de points de vue les traits féminins, que ce prêtre tire ses pouvoirs, comme c'est de l'union entre deux familles qu'un groupe peut espérer se reproduire. A dire vrai, le chamane apparaît comme un intermédiaire entre les hommes et les esprits : une fois l'alliance entre Mha et Shyangpo rompue par ce membre malencontreusement brisé (la symbolique de l'os reste toujours présente...), seul le chamane conserve la faculté de voir les esprits invisibles. Symboliquement parlant, l'alliance que "* prêtre contracte dans le monde surnaturel est d'ordre matrimonial. Alors que le prêtre clanique gère les relations de filiation à l'intérieur d'un cadre agnatique défini, le chamane s'apparente aux utérins. Les unions entre humains et celles entre esprits et humains sont conçues comme homologues. Les ordres social et religieux se confondent sous l'angle de la structure.

\section{L'os et la chair réunis : le cas magar}

Récapitulons. Les relations prêtre clanique-chamane sont en grande partie déductibles du couple parenté de l'os parenté de la chair. Le chamane est intimement lié aux cultes féminins, il prend en charge la part féminine (symbolisée chez les Tamang par la fleur) ${ }^{17}$ qui réside dans chaque

17. Jarmi les populations tribales du sous-continent indien, l'élément féminin qui s'oppose á l'os est aussi qualifí métaphoriquement de neur: cf. M. Carrin-Boue\% 1986 ). 
homme, tout particulièrement chez ces ètres androgynes que sont les enfants. Il marque l'intrusion des valeurs du sexe opposé dans une vie sociale que domine la patrilinéarité et qu'incarne, majoritairement, le prêtre clanique. Il est le symbole vivant de l'altérité.

Il existe cependant une exception de taille a cette bipolarisation religicuse. C'est celle des Magar, une population de quelque 210000 personnes selon les statistiques officielles de $1981^{18}$, qui vivent pour l'essentiel au sud du Dhaulagiri, dans le Népal occidental. Chez ceux de la Kali Gandaki, de Palpa et de Gulmi, on trouve par exemple un lien très fort entre les divinités lignagères kuläin, bhitre-kul ou kuldevatä et le chamanisme. Ces divinités (distinguées des pilri, "ancêtres " proprement dits, qui se réduisent à peu de (hoses) sont vénérées une fois l'an : soit à la pleine lune de Baiśākh, avril-mai, soit à celle de Mañsir, novembredécembre, r'est-à-dire au printemps ou à l'automne, selon les rlans. C'est un chamane -- appelé soit lama, soit jhãkriqui officie. La cérémonirs se déroule de nuit chez l'aîné du groupe de parenté, car c'est chez lui que sont gardés les dieux ancestraux kuläin. Elle rassemble tous les hommes adultes du groupe de patrifiliation, les femmes sont prosrrites. Le chamane entonne des chants religieux et entre en transe. Les kuläin parlent par sa bouche et fixent l'heur. du sarrifice. Un animal rest alors égorgé, son sang offert aux dieux-ancêtres et sa chair consomméc par les hommes au cours d'un repas communicl. Les rituels sont axés sur le poteau central de la maison auquel on accroche une gourde remplie de graines et autour duquel le chamane effectur plusicurs circumambulations avec les membres du clan. Lo: gendre a un rôle essenticl : c'est lui qui présente les offrandes

1×. (if. Populalion Census 1981 ip. 244 sq. - Ce chiffe doit ettre manie avec précaution et mériterait surement d'ètre récvalué. Les sources officielles népalaises ne considerent en effet que le critère de la langue maternelle et non l'idenlité ethnique. Or de nombreux Magar qui ont perdu aujourd'hui leur langue comtinuent de revendiquer cet elhnonyme. 
au kulāin et qui porte le matériel de culte. Une lampe à huile symbolisant l'ancêtre reste allumée toute la nuit ${ }^{19}$. Il n'est pas impossible que ces kuldevatā magar soient d'anciennes divinités villageoises, locales, adoptées progressivement par des clans particuliers (K. B. Bista, 1972, 128). L'un deux (parfois imaginé comme un couple), Maștā ou Maștā Barāha, évoque du reste la grande divinité du même nom des Indo-Népalais de l'Ouest, propitiée lors de fêtes villageoises et associée à des phénomènes oraculaires ${ }^{20}$. Quoi qu'il en soit, les cultes lignagers de ces Magar possèdent clairement une dimension ancestrale. Maștā Barāha, qui appartient à plusieurs clans, symbolise explicitement d'anciens aïeux, pitri, divinisés et apparaît souvent dans les rêves sous forme d'ancêtres ${ }^{21}$. On notera cependant que les dieux lignagers sont parfois considérés comme des esprits de parents décédés de façon anormale, bāyu, des morts dangereux en d'autres termes qui ont été pacifiés ultérieurement. Cette association avec la malemort pourrait aussi expliquer l'intervention de chamanes dans le culte.

Mais c'est parmi les Kham Magar, une population agropastorale d'environ 30000 personnes regroupées au sudouest du Dhaulagiri, dans des villages situés à une altitude supérieure à $2000 \mathrm{~m}$, que la configuration apparaît la plus particulière. Au sein de cette société, proche par sa vie matérielle des autres populations tibéto-birmanes du Népal, il n'y a pas division du champ sacerdotal. Le chamane (rammā ou jhãkri) est le seul spécialiste du groupe, l'unique porte-parole sur terre des esprits. De prêtre clanique il n'existe pas; quant aux chefs de village, leurs fonctions religieuses semblent minimes. Le rôle du jhĩkri est en fait

19. Cf. C. Jest (1976, 301-302), ainsi que ses rapports de mission c.xrs-ER, 299 (1986-1987), Gulmi-Argha Khanchi, dacty. Voir aussi J. Hitchcock (1976, 177) qui signale chez les Magar de la Bhujel Khola un lien particulièrement fort entre chamane et divinités claniques.

20. A ce sujet, voir le dossier présenté par M. Gaborieau (1969).

21. Communication personnelle de Marie Lecomte-Tilouine, qui prépare une thèse sur les Magar du district de Gulmi. 
cardinal dans la société kham magar et leur pouvoir apparaît étroitement associé à l'instance légitime du groupe: social. Contrairement à la plupart des chamanes népalais, qui possèdent un aspect marginal, volontiers sauvage, imprévu, le chamane procède ici d'une institution de type collégial qui " est à la fois structurellement inscrite dans l'organisation sociale et fonctionnellement contrôlée par elle " (de Sales, 1989, 121). Ce chamane en vérité est doublement défini au plan symbolique : il est non seulement le mari des esprits de la forêt, comme dans le cas magar décrit plus haut, mais aussi le fils d'un ancêtre, le plus souvent en ligne patrilinéaire. Il cumule de la sorte une relation d'alliance aux esprits et une relation de filiation agnatique.

On insistera ici davantage sur le second point qui semble spécifique à cette population. A quoi est attribuée la vocation du jeune chamane? Généralement au grand-père paternel, quelquefois au grand-père ou à l'oncle maternel. Le processus d'identification débute par une transe incontrôlable due à l'esprit du chamane décédé. De tels esprits sont particulièrement impatients de renaitre et reviennent souvent dans le monde des vivants dès la première année du deuil. Si l'élection du futur intercesseur est héréditaire, personne ne peut prévoir sur quelle personne elle se portera dans la famille, ni à quelle date exacte elle surviendra. L'esprit de l'ancêtre, $g$ l $^{\mathbf{2 2}}$, se manifeste dans le corps de l'élu lors de la séance chamanique. Les transes se reproduisent fréquemment, provoquant des désordres du comportement et un rétrécissement général des fonctions de relations : le néophyte ne mange plus, il tombe dans une apathie profonde, sa vie est en danger. Un maitre chamane est alors convoqué pour identifier l'ancêtre qui tourmente l'élu. Il pacifie l'esprit, le fait parler, le nomme. Il l'enferme, lui et ses alliés (des animaux de la forêt), dans une calebasse.

22. A. de Sales (1985, 95-97) donne également le mot barã pour désigner cet esprit ancestral. L'esprit résiderait dans le bas-ventre de l'homme. 
De ce jour, le néophyte appartient à son parent décédé, il lui est promis. Le pot n'est débouché que le jour de la grande initiation bosinya au cours de laquelle l'ancêtre et le nouveau chamane renaissent ensemble. A. de Sales (1985, 125), l'ethnologue sur laquelle nous nous fondons, précise: que la montée initiatique au mât que le jeune intercesseur doit accomplir pendant cette cérémonie symbolise son mariage avec l'esprit féminin de la forêt et que le cœur de bélier qu'il tient en haut de cet arbre représente l'ancêtre. Les deux aspects du chamane se trouvent ici conjoints.

D'autres groupes tribaux népalais ont tendance, il est vrai, à inscrire la vocation chamanique dans des liens héréditaires. Mais cette filiation passe chez eux davantage en ligne maternelle et il lui manque cette régularité quasi institutionnelle caractéristique des Kham Magar. Chez ces derniers en somme, il y a conjonction de la filiation et de l'alliance, de la parenté de l'os et celle de la chair (ou dans le cas présent du lait) dans la même personne. Dès lors, on ne s'étonnera pas que le chamane kham magar soit parfois amené à entrer en contact avec des unités patrilinéaires et à célébrer des rituels qui reviendraient ailleurs au prêtre clanique. C'est le cas en particulier d'une fête communale appelée Rãkya qui se tient le premier jour de Sāun, en juillet-août. Au cours de cette fête, le village expulse les mauvais esprits et redéfinit les limites de son territoire. Or, si dans un premier temps les villageois agissent seuls, ils sont bientôt secondés par des chamanes qui, grâce à leur bâton magique et aux battements de tambour que font résonner leurs assistants, chassent de chaque maison les âmes du monde d'en dessous. Mieux encore, A. de Sales (1989, 113-115) nous apprend que chaque lignage exogame délègue son chamane pour célébrer le rituel. Si le lignage ne compte pas d'intercesseur parmis ses membres, il doit en "louer " un pour l'occasion à un autre groupe de parenté et lui fournir les poulets et l'alcool dont il aura besoin pour l'exorcisme. A la fin, les chamanes sont invités à manger chez un notable 
du village avant de danser sur la place publique. Puis chaque lignage invite son intercesseur attitré pour procéder à des divinations pendant la nuit.

Comment comprendre ces chamanes domestiqués par des patrilignages? Deux hypothèses peuvent ètre envisagées. Soit les Kham Magar ont mieux que d'autres au Népal préservé une très ancienne tradition chamanique proche à beaucoup d'égards du chamanisme sibérien (centralité du jhãkri, mariage avec l'esprit féminin de la forêt, filiation patrilinéaire et valeurs agnatiques bien affirmées dans la figure du prêtre ${ }^{23}$. Soit un tel état exceptionnel de conservation résulterait d'un développement particulier. Faut-il y voir un vestige ou un faux archaïsme ?

La seconde hypothèse s'appuie sur des faits historiques précis : la conquête du Népal au xviII e siècle par Prithivi Narayan Shah, roi de Gorkha, et l'édification d'un Etat national au début $\mathrm{du} \mathrm{xIx}^{\mathbf{e}}$ siècle sous l'égide d'une dynastie hindoue. Ces événements entraînèrent une hindouisation, ou pour mieux dire une népalisation, des diverses populations du pays et une recomposition totale du paysage ethnique et social. La naissance d'un tel Etat centralisé balaya notamment les structures politiques traditionnelles basées sur de petites chefferies. On peut penser que les prêtres claniques des zones tribales perdirent petit à petit leurs attributs symboliques et dans certains cas disparurent. Cette explication repose implicitement sur l'idée que la bipolarisation des spécialistes religieux correspond à un fait très ancien parmi les populations tibéto-birmanes des collines népalaises : autant que le chamane, le prêtre clanique et le culte des ancêtres appartiendraient à la protohistoire des religions himalayennes. C'est ce que défend, entre autres, Allen (1976b, 526) quand il écrit : "Le culte des ancêtres est devenu de

23. Les chamanes kham magar ne sont-ils pas les seuls à porter des pantalons (y compris les chamanesses) ? Tous les autres chamanes népalais portent une jupe. Précisons que certains clans kham magar affirment venir originellement de régions sibériennes (Oppitz, 1983, 200). 
plus en plus périphérique à mesure que la société des castes s'est infiltrée dans les villages tribaux autrefois segmentés sur la base du territoire, que les relations temporaires et contractuelles ont supplanté les liens héréditaires, et que la langue nationale s'est imposée au détriment des langues locales." Ce dont les prêtres hindous et bouddhistes ont bénéficié, les chamanes ont pu eux aussi en tirer parti. Cependant, on s'explique mal, dans cette hypothèse, pourquoi la " népalisation " aurait eu aux $\mathrm{XvII}^{\mathrm{e}}-\mathrm{xIX} \mathrm{e}^{\mathrm{e}}$ siècles des effets si prononcés dans une région reculée comme celle des Kham Magar, alors que son impact fut moindre chez les Magar du Sud et les Gurung pourtant plus proches des centres hindous et davantage en contact avec les populations d'origine indienne. Force est de constater que des facteurs endogènes, dont on ne peut guère reconstituer l'enchaînement en l'étal actuel des recherches, jouèrent un rôle déterminant.

La première hypothèse insiste, elle, sur les difficultés d'accès du pays kham magar, difficultès qui auraient permis à cette région de rester à l'écart des royaumes hindous de l'ouest. Soyons clair : un tel isolement est illusoire. Il suffit de considérer la terminologie de parenté et la mythologie kham magar, plus imprégnées de termes népali que celles des Gurung et des Tamang, pour s'en convaincre. Pourtant, cette explication alternative peut aussi se prévaloir d'une dimension historique que personne ne s'est avisé jusqu'à présent de mettre en lumière. Réflexion faite, la "népalisation "dont le pays tire sa physionomie actuelle s'est effectuée de deux manières différentes : elle a été beaucoup plus violente et répressive à l'est qu'à l'ouest. Les tribus orientales telles les Limbu et les Rai ont lutté pied à pied contre les armées indo-népalaises de Prithivi Narayan Shah venues de l'ouest. Elles furent réduites militairement et intégrées de force, sans contrepartie, à l'Etat naissant. Cette défaite s'accompagna d'une pénétration en profondeur de leur territoire par des populations hindoues étrangères qui imposèrent leur culture et accaparèrent les meilleures terres. Les 
tribus de l'ouest, Gurung et Magar, en revanche, ne connurent pas ce traumatisme. Elles nouèrent dès le début une alliance avec le pouvoir Gorkha, s'engageant même comme mercenaires dans leurs troupes. En retour, les Gurung et les Magar bénéficièrent de nombreux avantages et purent négocier leur intégration. On peut par conséquent estimer que la conquête Shah a ébranlé de manière beaucoup plus radicale les structures claniques anciennes des tribus orientales que celles des tribus de l'ouest. Une telle reconstitution laisse dans l'ombre de nombreuses questions, notamment celle du prêtre clanique, mais elle aide à mieux cerner la permanence d'un chamanisme très archaïque en pays magar.

Elle permet également d'expliquer les aspects sauvages, paroxystiques du chamane limbu. Chez ces derniers en effet, les enfants qui présentent des signes de vocation chamanique sont possédés non par des ancêtres, mais par dess esprits maléfiques et terrifiants, tels ces esprits de la forêt ban jhãkri. La crise initiatique de ces jeunes gens prend une forme d'une violence extrême et les soustrait momentanément aux règles sociales : la personne s'enfuit dans la forêt voisine, vit comme un animal, reste prostrée, l'esprit ravi, dans un état de stupeur hébétée (Sagant, 1976). C'est, dit-on, ban jhîkri qui les a saisis et attirés dans les bois aver les battements de son tambour magique. Pour sortir de cet état cataleptique et devenir un chamane accompli, une procédure d'identification et d'apprentissage doit être envisagée sous la direction d'un guru, comme chez les Kham Magar. Mais parfois l'apprentissage avec un maître chamane s'avère superfétatoire : le néophyte reçoit directement son savoir des esprits surnaturels qui le possèdent. Ces chamanes, appelés mule, sont renommés pour être plus puissants que les autres. Les signes de la vocation apparaissent chez cux très tôt, dès la prime enfance (position du cordon ombilical par exemple), et déterminent rapidement un comportement asocial (ibid., 1976).

Ne peut-on voir dans ces traits imprévisibles des chamanes 
limbu le signe d'une désagrégation des structures sociopolitiques traditionnelles ? les intercesseurs dont la pratique: religieuse évolue vers des formes de possession et qui exercent en dehors des instances légitimes du groupe ne se seraient-ils pas marginalisés progressivement par suite d'une "népalisation "forcée et de l'incorporation du Népal oriental à une: formation étatique centralisée? De fait, tout semble indiquer que le chamanisme fut rejeté à la périphérie et se colora de valeurs féminines dans une très grande partie du Népal, notamment à l'est, alors qu'il resta central it attaché aux valeurs agnatiques chez les Magar. D'autres facteurs, telle la pénétration d'une religion dominante à tradition écrite comme le lamaïsme (absente chez les Kham Magar), jouèrent probablement le mème rôle chez les Tamang et les Gurung selon un mécanism» déjà décrit en Sibérie (Hamayon, 1982). D'êtres polyvalents au départ, les chamanes se seraient en somme: progressivement spécialisés dans la face nocturne et féminine; de l'univers, se chargeant d'une altérité foncière par rapport. a lrur voration première.

\section{Cionclusion}

Ainsi donc la dichotomie entre prètres claniques et chamanes choisic ici comme fil directeur serait l'aboutissement d'un long processus impulsé par l'édification d'un puissant. Etat national. La géographie du chamanisme au Vépal, aver: ce contraste si prononcé entre les ethnies de l'ouest comme les Magar et celles de l'est comme les Limbu et les Rai, traduirait des phénomènes historiques. ('est la raison pour laquelle on peut repérer parmi les populations de l'est et du centre de nombreuses interférences entre les drux grandes aatégories de représentations, liées l'une à la parenté agnatique, l'autre à la parenté utérinc. Chez les 'Tamang par exemple, qui occupent à bien des égards une position médiane entre les deux pòles magar et rai-limbu, les dieux du sol, apparentés au prêtre lambu, posièdent aux aussi une 
composante féminine et sont imaginés comme un couple. Inversement, le chamane, quel qu'il soit, est d'une certaine manière toujours associé aux valeurs de la masculinité et de l'agnation. Les Tamang en font le fils d'un ancêtre mort, même si le bompo peut difficilement rivaliser avec le prêtre clanique en matière d'ancestralité. Lorsque les deux types de prêtres coexistent, il y a d'ailleurs souvent cumul des fonctions sacerdotales dans la même personne. Le prêtre clanique: entre parfois en transe et part, comme le chamane, à la recherche des âmes égarées. Ne perdons pas de vue également que le chamane soigne autant les hommes que les femmes et que, même chez les Kham Magar, il peut être choisi dans certains ras en ligne utérine.

Quoique bien réels et fonctionnellement opératoires, les deux pôles ne sont donc pas totalement séparés. Ils comprennent toute une série de configurations intermédiaires entre lesquelles on pourrait répartir les sociétés des collines népalaises selon leurs caractéristiques propres. De quelque côté qu'on les envisage, la parenté de l'os et celle de la chair sont souvent imbriquées et admettent des points de contact. Le chamane notamment brouille les frontières : il concentre en lui les attributs des deux sexes, ceux des morts et des vivants. Toutes les lignes de partage se dissolvent dans la sphère qui est de son ressort. C'est que, comme ailleurs, la pensée religieuse de ces populations traditionnelles procède plus par inédiations que par oppositions bien tranchées. Le sacré ne se définit-il pas comme le lieu de toutes les ambiguïtés, de toutes les métamorphoses du même et de l'autre ?

Evoquons en guise de conclusion un dernier rapprochement. On a à maintes reprises établi un lien entre le chamane et la chasse. Dans ses rituels, le chamane piège les démons, ruse avec les esprits, traque l'âme des morts à la façon d'un chasseur poursuivant son gibier dans des territoires aussi inconnus que dangereux. On sait par ailleurs que le chamanisme le plus " pur " est né dans des sociétés où les activités cynégétiques (centrées sur la chair et le sang) jouent un rôle 
important. Mais on n'a guère attiré l'attention sur le lien tout. aussi fort qui existe entre le prêtre clanique et le pastoralisme. $\mathrm{Au}$ cours des cérémonies claniques, le lambu tamang retracr l'itinéraire suivi par ses ancêtres pour venir s'installer dans le village actuel. Or, bien qu'elle déborde du terroir proprement dit, cette liste recoupe les lieux-dits étagés selon l'altitude par lesquels passent les éleveurs quand ils montent en estive ou redescendent au village avec leurs troupeaux. Il est certes difficile d'établir une relation directe entre ces deux ordres de fait, mais la convergence s'impose ${ }^{24}$. Les fonctions symboliques du chamane et du prêtre clanique s'enracinent en fait fermement dans les modes de vie économiques. Comme l'a suggéré R. Hamayon (1989, 64-65) à propos de l'Asie septentrionale, la vie de chasse et le pastoralisme relèvent de deux logiques différentes : dans le premier cas domine une "logique de l'alliance" (sous forme de l'aide de l'allié rabatteur et du partage du gibier), dans le second prime une "logique de la filiation " basée sur l'utilisation et la transmission entre soi des troupeaux et des droits de pâturages. Malgré de nombreux glissements et transformations, que peuvent expliquer çà et là les évolutions historiques, l'opposition entre ancêtres claniques et esprits féminins occupe par conséquent une place fondamentale tant dans les systèmes d'idées que dans les activités quotidiennes des populations himalayennes. Elle s'inscrit dans un espace bipolaire, tendu entre filiation et alliance, entre élevage et chasse, à l'intéricur duquel évolue la pensée symbolique.

24. A propos des Kham Magar, M. (Jppitz (1983, 212, remarque (en s'en donnant) que le chamane est tenu a l'écart des rites de passage dans lesquels cette dimension itinerante du pastoralisme intervient. Notons cependant que le chamane tamangr récite lui aussi, lors de son voyağe à la recherche des âmes, une série de noms de lieux qui inclut ('i-Same (Samye) au Tibet, le lieu d'origine mythique de ce groupe ethnique. 
OLVRAGES CITÉS

Allen, V. J. (1975), Byansi kinship terminology. A study in symmetry, Man (n.s.), vol. 10, 1, 80)-94.

Allen, N. J. (1976a), Studies in the myths and oral traditions of the Thulung Rai of East Nepal, Oxford, Linacre College, Ph.D., dact.

Allen, N. J. (1976b), Approaches to illness in the Nepalese hills, in Social Anthropology and Medecine, ed. by J. B. London, LondonNew York-San Francisco, Academic Press, p. 500-552.

Allen, N. J. (1981), The Thulung myth of the Bhume sites and some indo-tibetan comparisons, in Asian Highland societies in Anthropological perspective, ed. by C. von Fürer-Haimendorf, New Delhi, Sterling Publishers, p. 168-182.

Bista, K. B. (1972), Le culte du Kuldevata au Népal, en parliculier chez certains Ksatri de la vallée de Kathmandou, Paris, Ed. du cNrss.

Currin-Bouez, M. (1986), La fleur et l'os. Symbolisme et rituel chez les Santal, Paris, Ed. de l'Ecole des Hautes Etudes en Sciences sociales (coll. "Cahiers de l'Homme").

Dollfus, P. (1989), Lieu de neige et de genécriers. Organisation sociale et religieuse des communautés bouddhistes du I.adakh, Paris, Ed. du CNRs (coll. "Ftudes himalayennes").

Fournier, A. (1974), The role of priest in Sunuwar society, Kailash, vol. II, 3, 153-166.

Gaborieau, M. (1969), Notes préliminaires sur le dieu Maștāa, Objets et mondes, t. IX, 1, 19-50.

Gorer, G. (1938), Himalayan village. An Account of the Lepchas of Silikim, London, M. Joseph.

Hamayon, R. (1982), Des chamancs au chamanisme, L'Ethnographie, t. $87-88,2-3,13-48$.

Hamayon, R. (1989), Religions de l'Asie septentrionale, Annuaire. Résumé des Conférences et Travaux EPHE. Section des Sciences religieuses, t. XCVI, 1987-1988, 64-69.

Hitchcock, J. T. (1976), Aspects of Bhujel shamanism, in Spirit possession in the Nepal Himalayas, ed. by .J. 'T. Ilitchcock and R. L. Jones, Warminster, Aris \& Phillips, p. 165-196.

Höfer, A. (1974), Is the bombo an ecstatic ? Some ritual techniques of Tamang shamanism, in Contributions to the Anthropology of Nepal, ed. by C. von Fürer-Haimendorf, Warminster, Aris \& Phillips, p. 168-182.

Höfer, A. (1981), Tamang rilual texts. I. Preliminary sludies in the folli-religion of an ethnic minority in Nepal, Wiesbaden, Franz Steiner Verlag.

Holmberg, D. H. (1981)), Lama, shaman and lambu in Tamang religious practice, Cornell University, Ph.D. 
Holmberg, D. H. (1983), Shamanic soundings : Femaleness in the Tamang ritual structure, Journal of Women in Culture and Society, vol. $9,1,40-58$.

Holmberg, D. H. (1989), Order in paradox. Myth, ritual and exchange among Nepal's Tamang, Ithaca-London, Cornell University Press.

Jäschke, H. A. (1949), Tibelan-English diclionary, London, Routledge \& Kegan Paul.

Jest, C. (1975), Dolpo. Communautés de langue libétaine du Népal, Paris, Ed. du cinrs.

Jest, C. (1976), Encounters with intercessors ill Nepal, in spirit possession in the Nepal Himayalas, ed. by J. $\mathrm{T}$. Ifitchcock and R. L. Jones, Warminster, Aris \& Phillips, p. 294-308.

Jest, C. (1986-1987), Rapports de mission Gulmi-Argha Khanchi, paris, CNRS (UPR 299), dact.

Kvaerne, P. (1981), Mythes cosmogoniques au Tibet, in Dictionnaire des mythologies, sous la dir. de Y. Bommefoy, Paris, Flammarion, p. 249-252.

Lévi-Strauss, C. (1967), Les structures élémentaires de la parenté, Paris-La Haye, Mouton (1re ed., 1947).

Levine, N. E. (1988), The Dynamics of Polyandry. Kinship, domesticity and population on the Tibelan border, Chicago, The University of Chicago Press.

Mac Dougal, C. (1973), Structure and division in Kulunge Rai society, Kailash, vol. I, 3, 205-224.

Meyer, F. (1981), Gso-ba Rig-pa. Le systeme médical tibétain. P'aris, Ed. du cors (coll. " (iahiers népalais").

Oppitz, M. (1983), The wild boar and the plough. Origin stories of the Northern Magar, Kailash, vol. X, 3-4, 187-226.

Oppitz, M. (1988), Frau für fron. Die Dreierallianz bei den Magar West-Nepals, Frankfurt am Main, Suhrkamp.

Padoux, A. (1984), Le monde hindou et le sexe : symbolisme, attitudes, pratiques, Cahiers internationaux de sociologie, vol. LXXVI, 29-49.

Pignède, B. (1966), Les Gurungs, une population himalayenne du Népal, Paris, Mouton \& Co.

Population Census, 1981. Geographic Region Tables. Vol. II, Kathmandu, wnc, Central Bureau of Statistics, 1984.

Sagant, P. (1976), Becoming a Limbu priest. Ethnographic notes, in Spirit Possession in the Nepal Himalayas, ed. by .J. T. Hitchcock and R. L. Jones, Warminster, Aris \& Phillips, p. 56-99.

Sagant, P. (1982), I,e chamane et la grêle, L'Ethnographie, t. 87-88, $2-3, \quad 163-174$.

Sales, A. de (1985), Acles el paroles dans les riluels chamaniques des Kham Magar (Népal). thèse de doctorat, Universiti de Paris $\mathrm{X}$, dact.

Sales, $A$. de (1989), Gendres des exprits : les chamanes Kham-Magar, in Prètrise, pouvoirs al aulorité en Himalaya, eds V. Bouillier 
and G. Toffin, Paris, Editions de l'Ecole des Hautes Etudes en Sciences sociales, p. 101-125 (coll. "Purușārtha ").

Stein, R. A. (1962), La civilisation tibétaine, Paris, Dunod.

Steinmann, B. (1987a), Les Tamang du Népal. Usages et religion, religion de l'usage, Paris, Ed. Recherches sur les Civilisations, ADPF.

Steinmann B. $(1987 b)$, Le culte des dieux du clan chez les Tamang: la terre, le livre et la lignée, L'Ethnographie, t. 83, 100-101, 303-328.

Strickland, S. S. (1982), Beliefs, practices, and legends. A study in the narrative poetry of the Gurungs of Nepal, Cambridge, Jesus College, Ph.D., dact.

Strickland, S. S. (1983), The Gurung priest as bard, Kailash, vol. X, 3-4, 227-265.

Tofrin, G. (1986), Unités de parenté, système d'alliance et de prestatious chez les Tamang de l'Ouest (Népal), Anthropos, vol. 81, 21-45.

loffin, G. (1987), Dieux du sol et démons dans les religions himalayennes, Etudes rurales, no 107-108, 85-106.

Toffin, G. (1990), Compte rendu de N. E. Levine, The Dynamics of Polyandry (1988), in L'Homme, sous presse.

Toffin, G., Jest, C. and Blamont, D. (1986), Les populations de la région Ankhu Khola-Trisuli, in Les collines du Népal central, éd. J. F. Dobremez, Paris, Ed. de l'inra, t. 1, p. 79-118. 